\title{
Asymptotic Inference for the Weak Stationary Double AR(1) Model
}

\author{
Fang Chang, Augustine C. M. Wong*, Yanyan Wu \\ Department of Mathematics and Statistics, York University, Toronto, Ontario, Canada \\ Email: \{changf, ${ }^{*}$ august, minna\}@yorku.ca
}

Received November 11, 2011; revised December 15, 2011; accepted December 30, 2011

\begin{abstract}
An AR(1) model with ARCH(1) error structure is known as the first-order double autoregressive (DAR(1)) model. In this paper, a conditional likelihood based method is proposed to obtain inference for the two scalar parameters of interest of the DAR(1) model. Theoretically, the proposed method has rate of convergence $O\left(n^{-3 / 2}\right)$. Applying the proposed method to a real-life data set shows that the results obtained by the proposed method can be quite different from the results obtained by the existing methods. Results from Monte Carlo simulation studies illustrate the supreme accuracy of the proposed method even when the sample size is small.
\end{abstract}

Keywords: Canonical Parameter; Double Autoregressive Model; $p$-Value Function; Signed Log-Likelihood Ratio Statistic

\section{Introduction}

ARCH error structure was first introduced to econometric models by Engle [1] as a way of unleashing the constant variance assumption. Weiss [2] studied the AR models with ARCH error structure. A special case of Weiss [2] model is the AR(1) model with ARCH(1) error structure, which is also known as the first-order double autoregressive (DAR(1)) model. The DAR(1) model can be expressed as:

$$
Y_{0}=\eta_{0}, \quad Y_{t}=\phi Y_{t-1}+\eta_{t} \sqrt{\omega+\alpha Y_{t-1}^{2}}, \quad t=1, \cdots, n
$$

where $\omega, \alpha>0$ and $\eta_{0}, \cdots, \eta_{n}$ is a sequence of independent standard normal random variables. Then

$\operatorname{var}\left(Y_{0}\right)=1$ and $\operatorname{var}\left(Y_{t} \mid Y_{t-1}\right)=\omega+\alpha Y_{t-1}^{2}, \quad t=1, \cdots, n$, which is varying over time. This nonlinear time series model is also a special case of $\beta$-ARCH model investigated in Guégan \& Diebolt [3] with $\beta=1$. This type of model is widely used for fitting financial time series data because the influence of the empirical observations is part of the source for volatility. Guégan \& Diebolt [3] derived the sufficiency condition for the weak stationary DAR(1) model to be $\phi^{2}+\alpha<1$, and Borkovec \& Klüppelberg [4] proved that it is also the necessity condition for the model. Figure 1 shows the weak stationary region for the DAR(1) model graphically. Note that, for the DAR(1) model, when $(\phi, \alpha)$ reaches the boundary points $( \pm 1,0)$, the model becomes a nonstationary $\operatorname{AR}(1)$

*Corresponding author. model.

Ling [5] obtained a conditional likelihood function for the weak stationary DAR(1) model. Then he derived the asymptotic distribution for the maximum likelihood estimate of the parameters based on this conditional likelihood function.

In Section 2, some asymptotic likelihood-based inference procedures for a general model are reviewed. In Section 3, a modified signed log conditional likelihood ratio statistic for the weak stationary DAR(1) model is derived. The proposed method, theoretically, has rate of convergence $O\left(n^{-3 / 2}\right)$. A real-life example is presented in Section 4 to illustrate the implementation of the proposed method and also to show that results obtained from the methods discussed in this paper can be quite different. Results from Monte Carlo simulation studies are also presented in Section 4 to illustrate the extreme accuracy of the proposed method even when the sample size is small. Some concluding remarks are given in Section 5 .

\section{Asymptotic Likelihood-Based Inference for a General Model}

Let $y^{0}=\left(y_{1}, \cdots, y_{n}\right)^{\prime}$ be a sample from a canonical exponential family model with log likelihood function $\ell(\theta)=\ell\left(\theta ; y^{0}\right)$, where $\theta=\left(\psi, \lambda^{\prime}\right)^{\prime}$ is the $k$-dimensional canonical parameter, with $\psi$ being the scalar parameter of interest and $\lambda$ being the $(k-1)$-dimensional nuisance parameter. Denote $\hat{\theta}=\left(\hat{\psi}, \hat{\lambda}^{\prime}\right)^{\prime}$ be the 


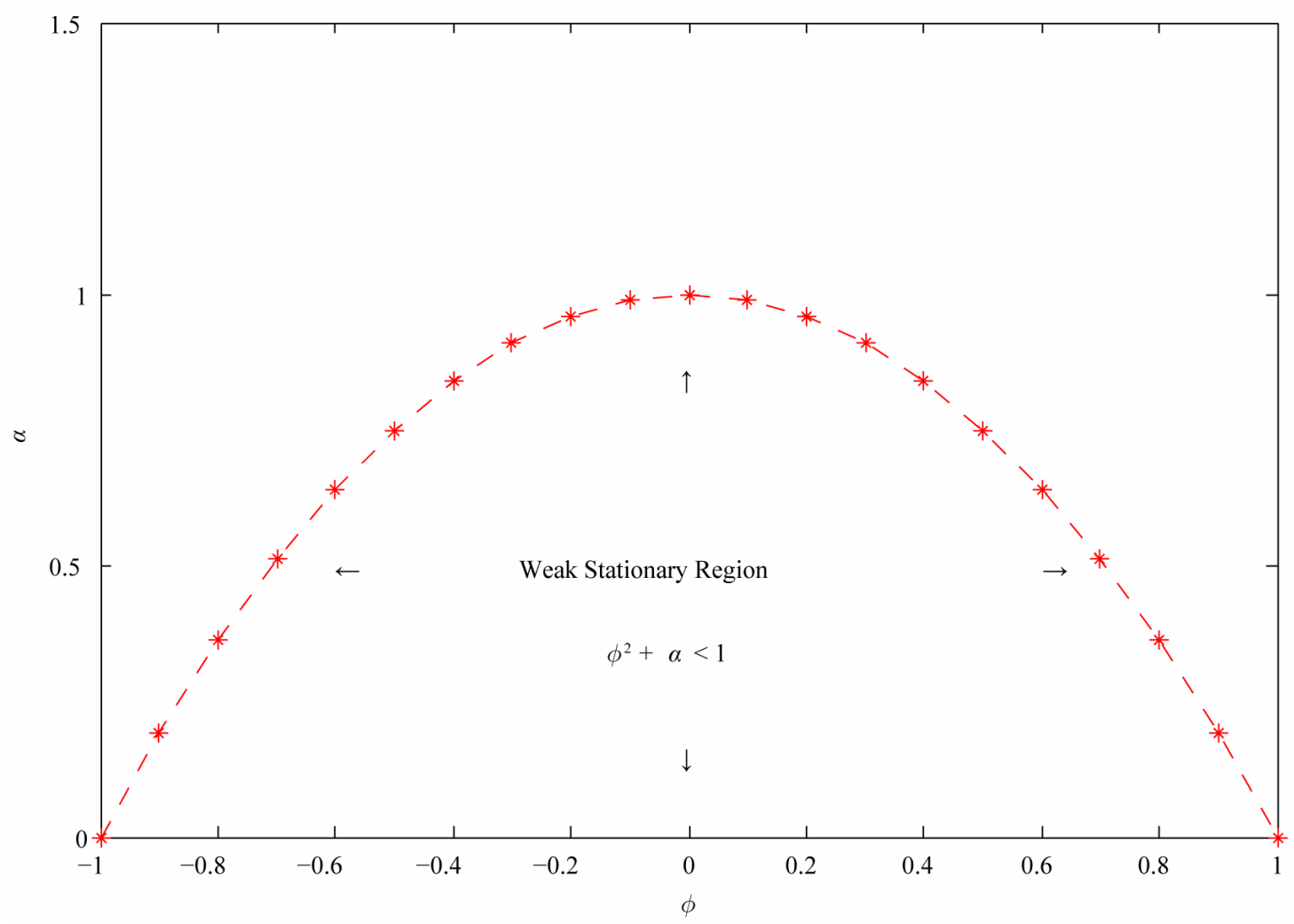

Figure 1. Weakly stationary region for DAR(1).

overall maximum likelihood estimate (MLE) of $\theta$, and an estimate of the variance of $\hat{\theta}$ is $j_{\theta \theta^{\prime}}^{-1}(\hat{\theta})$ where

$$
j_{\theta \theta^{\prime}}(\theta)=-\frac{\partial^{2} \ell(\theta)}{\partial \theta \partial \theta^{\prime}}
$$

is the observed information matrix. Moreover, let

$\hat{\theta}_{\psi}=\left(\psi, \hat{\lambda}_{\psi}^{\prime}\right)^{\prime}$ be the constrained maximum likelihood estimate of $\theta$ for a given $\psi$, and

$$
j_{\lambda \lambda^{\prime}}(\theta)=-\frac{\partial^{2} \ell(\theta)}{\partial \lambda \partial \lambda^{\prime}}
$$

is the nuisance observed information matrix.

With the regularity conditions stated in Wald [6], and a the scalar parameter of interest $\psi$, as $n \rightarrow \infty$, we have

$$
q \equiv q(\psi)=\frac{\hat{\psi}-\psi}{\sqrt{\widehat{\operatorname{var}(\hat{\psi})}}}
$$

is asymptotically distributed as standard normal and $\widehat{\operatorname{var}}(\hat{\psi})$ is the $(1,1)$ entry of $j_{\theta \theta^{\prime}}^{-1}(\hat{\theta})$. Thus, a $(1-\gamma) 100 \%$ confidence interval for $\psi$ based on (2) can be approximated by

$$
\left(\hat{\psi}-z_{\gamma / 2} \sqrt{\widehat{\operatorname{var}}(\hat{\psi})}, \hat{\psi}+z_{\gamma / 2} \sqrt{\widehat{\operatorname{var}(\hat{\psi})}}\right)
$$

where $z_{\gamma / 2}$ is the $(1-\gamma / 2) 100^{\text {th }}$ percentile of the standard normal distribution. Alternatively, the $p$-value function for $\psi$ can be approximated by $p(\psi)=\Phi(q)$, where $\Phi(\cdot)$ is the cumulative distribution function of the standard normal distribution. Note that $q(\psi)$ is the standardized maximum likelihood estimate departure in the canonical parameter scale.

Although the Wald method is simple to use, one of the major disadvantages is that the methodology is not invariant to reparameterization. Moroever, the Wald method does not take into consideration of the effect of the nuisance parameter. For a scalar parameter of interest, a familiar measure that is invariant to reparameterization is the signed log-likelihood ratio (SLR) statistic:

$$
r \equiv r(\psi)=\operatorname{sgn}(\hat{\psi}-\psi)\left\{2\left[\ell(\hat{\theta})-\ell\left(\hat{\theta}_{\psi}\right)\right]\right\}^{1 / 2} .
$$

With regularity conditions as stated in DiCiccio, Field \& Fraser [7], $r$ is also asymptotically distributed as the standard normal distribution. Hence, a $(1-\gamma) 100 \%$ central confidence interval for $\psi$ based on (3) can be approximated by

$$
\left\{\psi:|r(\psi)|<z_{\gamma / 2}\right\}
$$

and the corresponding $p$-value function for $\psi$ is $p(\psi)=\Phi(r)$.

It is well-known that both the Wald method and the 
SLR method are not very accurate especially when the sample size is small. Theoretically, they only have rate of convergence $O\left(n^{-1 / 2}\right)$. In statistics literature, there exists other methods that have higher rate of convergence. In particular, Barndorff-Nielsen ([8,9]) derived a modified signed log-likelihood ratio statistic for any scalar parameter $\psi$ :

$$
r^{*} \equiv r^{*}(\psi)=r(\psi)+\frac{1}{r(\psi)} \log \left\{\frac{Q(\psi)}{r(\psi)}\right\}
$$

and proved that it is asymptotically distributed as the standard normal distribution with rate of convergence $O\left(n^{-3 / 2}\right)$, where $r(\psi)$ is the signed log-likelihood ratio statistic as defined in (3), and $Q(\psi)$ is a quantity that needs to be specifically defined for each model being considered. For the canonical exponential family model, and $\psi$ being a component parameter of the canonical parameter, $Q(\psi)$ takes the form of

$$
Q(\psi)=(\hat{\psi}-\psi)\left\{\frac{\left|j_{\theta \theta^{\prime}}(\hat{\theta})\right|}{\left|j_{\lambda \lambda^{\prime}}\left(\hat{\theta}_{\psi}\right)\right|}\right\}^{1 / 2}
$$

which is the standardized maximum likelihood departure in canonical parameter scale taking into consideration of removing the nuisance parameter. Hence a $(1-\gamma) 100 \%$ central confidence interval for $\psi$ based on $r^{*}(\psi)$ is

$$
\left\{\psi:\left|r^{*}(\psi)\right|<z_{\gamma / 2}\right\}
$$

and the corresponding $p$-value function for $\psi$ is $p(\psi)=\Phi\left(r^{*}\right)$.

However, not every model belongs to the canonical exponential family model, and even if it does, the parameter of interest may not be a component parameter of the canonical parameter. Fraser \& Reid [10] showed that any statistical model with a known density function depending on a natural parameter $\theta$ can be approximated by a tangent exponential model with a locally defined canonical parameter, $\varphi(\theta)$ :

$$
\varphi^{\prime}(\theta)=\left.\frac{\partial \ell(\theta)}{\partial y}\right|_{y^{0}} \times V
$$

where $V=\left.\frac{\partial y}{\partial \theta^{\prime}}\right|_{\left(y^{0}, \hat{\theta}\right)}$ is considered as the tangent direc-

tion. Differentiating the locally defined canonical parameter $\varphi(\theta)$ with respect to the natural parameter $\theta$, we have

$$
\varphi_{\theta^{\prime}}(\theta)=\frac{\partial \varphi(\theta)}{\partial \theta^{\prime}} .
$$

By changing the parameter space from $\theta$ to $\varphi(\theta)$, the maximum likelihood estimate departure $\hat{\psi}-\psi$ in $\theta$ scale is equivalent to $\operatorname{sgn}(\hat{\psi}-\psi)\left|\chi(\hat{\theta})-\chi\left(\hat{\theta}_{\psi}\right)\right|$ in the locally defined canonical parameter $\varphi(\theta)$ scale, where

$$
\chi(\theta)=\frac{\psi_{\varphi^{\prime}}\left(\hat{\theta}_{\psi}\right)}{\left|\psi_{\varphi^{\prime}}\left(\hat{\theta}_{\psi}\right)\right|} \varphi(\theta),
$$

with first factor is the unit row vector version of the gradient vector, which is obtained from

$$
\begin{aligned}
\psi_{\varphi^{\prime}}(\theta) & =\frac{\partial \psi(\theta)}{\partial \varphi^{\prime}}=\left(\frac{\partial \psi(\theta)}{\partial \theta^{\prime}}\right)\left(\frac{\partial \varphi(\theta)}{\partial \theta^{\prime}}\right)^{-1} \\
& =\psi_{\theta^{\prime}}(\theta) \varphi_{\theta^{\prime}}^{-1}(\theta) .
\end{aligned}
$$

Moreover, by the chain rule in differentiation, determinant of the observed information matrix in $\theta$ scale, $\left|j_{\theta^{\prime}}(\hat{\theta})\right|$, expressed in $\varphi(\theta)$ scale is

$$
\left|j_{\varphi \varphi^{\prime}}(\phi(\hat{\theta}))\right|=\left|j_{\theta \theta^{\prime}}(\hat{\theta})\right|\left|\varphi_{\theta}(\hat{\theta})\right|^{-2}
$$

and similarly, the determinant of the nuisance observed information matrix, $\left|j_{\lambda \lambda^{\prime}}\left(\hat{\theta}_{\psi}\right)\right|$, expressed in $\varphi(\theta)$ scale is

$$
\left|j_{\left(\lambda \lambda^{\prime}\right)}\left(\hat{\theta}_{\psi}\right)\right|=\left|j_{\lambda \lambda^{\prime}}\left(\hat{\theta}_{\psi}\right)\right|\left|\varphi_{\lambda^{\prime}}^{\prime}\left(\hat{\theta}_{\psi}\right) \varphi_{\lambda^{\prime}}\left(\hat{\theta}_{\psi}\right)\right|^{-1} .
$$

Hence $Q$ in $\varphi(\theta)$ scale can be expressed as

$$
\begin{aligned}
Q=Q(\psi)= & \operatorname{sgn}(\hat{\psi}-\psi)\left|\chi(\hat{\theta})-\chi\left(\hat{\theta}_{\psi}\right)\right| \\
& \times\left\{\frac{\left|j_{\theta \theta^{\prime}}(\hat{\theta})\right|\left|\varphi_{\theta^{\prime}}(\hat{\theta})\right|^{-2}}{\left|j_{\lambda \lambda^{\prime}}\left(\hat{\theta}_{\psi}\right)\right|\left|\varphi_{\lambda^{\prime}}^{\prime}\left(\hat{\theta}_{\psi}\right) \varphi_{\lambda^{\prime}}\left(\hat{\theta}_{\psi}\right)\right|^{-1}}\right\}^{1 / 2}
\end{aligned}
$$

Hence $r^{*}(\psi)$, which has rate of convergence $O\left(n^{-3 / 2}\right)$, can be obtained. Thus a $(1-\gamma) 100 \%$ confidence interval for $\psi$, and also the $p$-value function for $\psi$ can be obtained.

\section{A Modified Log Conditional Likelihood Ratio Statistic for the DAR(1) Model}

For the DAR(1) model given in (1), Ling [5] obtained the conditional likelihood function for the weak stationary DAR(1) model. Moreover, assuming $\omega$ is known, Ling $([5,11])$ studied the asymptotic distribution for the maximum likelihood estimate of $\theta, \hat{\theta}=(\hat{\varphi}, \hat{\alpha})^{\prime}$ based on the conditional likelihood function. More specifically, the log conditional likelihood function for DAR(1) model with $\omega$ known is:

$$
\begin{aligned}
\ell(\theta) & =\ell(\phi, \alpha)=\ell\left(\theta ; y^{0}\right) \\
& =-\frac{1}{2} \sum_{t=1}^{n}\left(\log \left(\omega+\alpha y_{t-1}^{2}\right)+\frac{\left(y_{t}-\phi y_{t-1}\right)^{2}}{\omega+\alpha y_{t-1}^{2}}\right) .
\end{aligned}
$$


Hence, $\ell_{\theta}(\theta)=\frac{\partial \ell(\theta)}{\partial \theta}=\left(\frac{\partial \ell(\theta)}{\partial \varphi}, \frac{\partial \ell(\theta)}{\partial \alpha}\right)$ where

$$
\begin{aligned}
& \frac{\partial \ell(\theta)}{\partial \phi}=\sum_{t=1}^{n} \frac{y_{t-1}\left(y_{t}-\phi y_{t-1}\right)}{\omega+\alpha y_{t-1}^{2}}, \\
& \frac{\partial \ell(\theta)}{\partial \alpha}=\frac{1}{2} \sum_{t=1}^{n} \frac{y_{t-1}^{2}}{\omega+\alpha y_{t-1}^{2}}\left(\frac{\left(y_{t}-\phi y_{t-1}\right)^{2}}{\omega+\alpha y_{t-1}^{2}}-1\right)
\end{aligned}
$$

and the overall MLE, $\hat{\theta}=(\hat{\varphi}, \hat{\alpha})^{\prime}$, can be obtained by solving

$$
\left.\frac{\partial \ell(\theta)}{\partial \varphi}\right|_{\theta=\hat{\theta}}=0,\left.\frac{\partial \ell(\theta)}{\partial \alpha}\right|_{\theta=\hat{\theta}}=0 .
$$

The observed information matrix $j_{\theta^{\prime}}(\theta)$ is (see the bottom of the page)

Ling [5] showed that the expectation of $\hat{\theta}$ is asymptotically equal to $\theta$, and instead of using $j_{\theta \theta^{\prime}}^{-1}(\hat{\theta})$ as the asympotitic variance for $\hat{\theta}$, he showed that

$$
\operatorname{var}(\hat{\theta}) \approx\left(\begin{array}{cc}
\left(n E \frac{Y_{t}^{2}}{\omega+\alpha Y_{t}^{2}}\right)^{-1} & 0 \\
0 & \frac{2 A}{n\left(A C-B^{2}\right)}
\end{array}\right),
$$

where

$$
\begin{gathered}
A=E\left\{\frac{1}{\left(\omega+\alpha Y_{t}^{2}\right)^{2}}\right\}, B=E\left\{\frac{Y_{t}^{2}}{\left(\omega+\alpha Y_{t}^{2}\right)^{2}}\right\}, \\
C=E\left\{\frac{Y_{t}^{4}}{\left(\omega+\alpha Y_{t}^{2}\right)^{2}}\right\}
\end{gathered}
$$

Since this asymptotic variance of $\hat{\theta}$ still involves the unknown parameter $\alpha$, Ling [5] further proposed to use

$$
\widehat{\operatorname{var}}(\hat{\theta})=\left(\begin{array}{cc}
\left(\sum_{t=1}^{n} \frac{y_{t}^{2}}{\omega+\hat{\alpha} y_{t}^{2}}\right)^{-1} & 0 \\
0 & \frac{2 \hat{A}}{\hat{A} \hat{C}-\hat{B}^{2}}
\end{array}\right),
$$

as an estimate of $\widehat{\operatorname{var}}(\hat{\theta})$, where

$$
\hat{A}=\sum_{t=1}^{n} \frac{1}{\left(\omega+\hat{\alpha} y_{t}^{2}\right)^{2}}, \quad \hat{B}=\sum_{t=1}^{n} \frac{y_{t}^{2}}{\left(\omega+\hat{\alpha} y_{t}^{2}\right)^{2}},
$$

$$
\hat{C}=\sum_{t=1}^{n} \frac{y_{t}^{4}}{\left(\omega+\hat{\alpha} y_{t}^{2}\right)^{2}} .
$$

Then inference concerning $\phi$, and $\alpha$ can be obtained by the Wald method.

For the DAR(1) model, Ling [5] pointed out that it is an ergodic process under suitable conditions on the parameters. Therefore, by Taylor expansion, we have

$$
\ell(\theta)-\ell(\hat{\theta}) \approx \frac{1}{2}(\theta-\hat{\theta})^{\prime} \ell_{\theta \theta^{\prime}}(\hat{\theta})(\theta-\hat{\theta}) .
$$

Hence

$$
-2[\ell(\theta)-\ell(\hat{\theta})] \approx \frac{1}{2}(\hat{\theta}-\theta)^{\prime} j_{\theta \theta^{\prime}}(\hat{\theta})(\hat{\theta}-\theta)
$$

is asymptotically distributed as a $\chi^{2}$ distribution with $p$ degrees of freedom. Thus, when $\operatorname{dim}(\theta)=p=1$, the signed $\log$ conditional likelihood ratio statistic for $\phi$ and for $\alpha$ of the DAR(1) model is asymptotically distributed as $N(0,1)$ with rate of convergence $O\left(n^{-1 / 2}\right)$.

We performed some simulation studies for testing the normality of the log conditional likelihood ratio statistics for the DAR(1) model. The Kolmogorov-Smirov test is employed. We considered a medium sample size of $n=50$ and a large sample size of $n=200$. For each sample size, we generate 10,000 samples from the DAR(1) model for each combination of parameter values. For each generated sample, the signed log conditional likelihood ratio statistic for $\phi$ and for $\alpha$ are calculated. The simulation results are presented in Tables $\mathbf{1}$ and 2: Table 1 records the $p$-values of the Kolmogorov-Smirov test when the parameters values are on the boundary of the weak stationary region; Table 2 records the $p$-values of the Kolmogorov-Smirov test when the parameters values are the interior points of the weak stationary region. From the tables, the $p$-values of the KolmogorovSmirov test of the signed log conditional likelihood ratio statistic for $\phi$ are large regardless of the sample sizes. Hence no evidence that the signed log conditional likelihood ratio statistic for $\phi$ is not distributed as $N(0,1)$. On the other hand, the $p$-values of the KolmogorovSmirov test of the signed log conditional likelihood ratio statistic for $\alpha$ are large only for $n=200$. Hence for $n$ is sufficiently large, there is no evidence that the signed $\log$ conditional likelihood ratio statistic for $\alpha$ is not distributed as $N(0,1)$.

Note that when $\omega$ is unknown, Ling \& Li [12] sug-

$$
\left.j_{\theta \theta^{\prime}}(\theta)=-\frac{\partial^{2}}{\partial \theta \partial \theta^{\prime}} \ell(\theta)=\left(\begin{array}{cc}
\sum_{t=2}^{n} \frac{y_{t-1}^{2}}{\omega+\alpha y_{t-1}^{2}} & \sum_{t=2}^{n} \frac{y_{t-1}^{3}\left(y_{t}-\phi y_{t-1}\right)}{\left(\omega+\alpha y_{t-1}^{2}\right)^{2}} \\
\sum_{t=2}^{n} \frac{y_{t-1}^{3}\left(y_{t}-\phi y_{t-1}\right)}{\left(\omega+\alpha y_{t-1}^{2}\right)^{2}} & \sum_{t=2}^{n} \frac{y_{t-1}^{4}}{2\left(\omega+\alpha y_{t-1}^{2}\right)^{2}}\left\{\frac{2\left(y_{t}-\phi y_{t-1}\right)^{2}}{\omega+\alpha y_{t-1}^{2}}-1\right.
\end{array}\right\}\right) .
$$


Table 1. The $p$-values of the Kolmogorov-Smirnov test for the SLR when $\omega=1, \phi$ and $\alpha$ are on the boundary of the weak stationary region.

\begin{tabular}{cccccc}
\hline$(\phi, \alpha)$ & $(-0.95,0.0975)$ & $(-0.5,0.75)$ & $(0,1)$ & $(0.5,0.75)$ & $(0.95,0.0975)$ \\
\hline$\psi=\phi(n=50)$ & 0.2093 & 0.3089 & 0.0923 & 0.2519 & 0.1676 \\
$\psi=\phi(n=200)$ & 0.7294 & 0.2544 & 0.2219 & 0.8769 & 0.3595 \\
$\psi=\alpha(n=50)$ & 0.0054 & 0.0184 & 0.0102 & 0.0155 & 0.0217 \\
$\psi=\alpha(n=200)$ & 0.2162 & 0.6260 & 0.5481 & 0.3084 & 0.7428 \\
\hline
\end{tabular}

Table 2. The $p$-values of the Kolmogorov-Smirnov test for the SLR when $\omega=1, \phi$ and $\alpha$ are interior points of the weak staionary region.

\begin{tabular}{|c|c|c|c|c|c|c|c|c|}
\hline$(\phi, \alpha)$ & $(-0.95,0.0975)$ & $(-0.5,0.5)$ & $(-0.5,0.25)$ & $(0,0.5)$ & $(0.1,0.4)$ & $(0.5,0.25)$ & $(0.5,0.5)$ & $(0.95,0.09)$ \\
\hline$\psi=\phi$ & 0.0647 & 0.0525 & 0.6512 & 0.3877 & 0.0614 & 0.4067 & 0.5246 & 0.9448 \\
\hline \multicolumn{9}{|c|}{$(n=50)$} \\
\hline$\psi=\phi$ & 0.1418 & 0.2234 & 0.2626 & 0.3933 & 0.9673 & 0.7956 & 0.6339 & 0.0996 \\
\hline \multicolumn{9}{|c|}{$(n=200)$} \\
\hline$\psi=\alpha$ & 0.0703 & 0.0021 & 0.0628 & 0.0001 & 0.1980 & 0.0000 & 0.0011 & 0.0013 \\
\hline \multicolumn{9}{|c|}{$(n=50)$} \\
\hline$\psi=\alpha$ & 0.2778 & 0.1640 & 0.0659 & 0.1865 & 0.1779 & 0.5938 & 0.0660 & 0.1802 \\
\hline \multicolumn{9}{|c|}{$(n=200)$} \\
\hline
\end{tabular}

gested a method to estimate $\omega$ and the analysis in Ling \& Li [12] treated the estimate $\omega$ as the known $\omega$. For the rest of the section, we following the approach of Ling \& Li [12] and derived the modified signed log conditional likelihood ratio statistic for $\phi$ and $\alpha$ respectively.

When the parameter of interest is $\psi(\theta)=\phi$, the nuisance parameter is $\lambda=\alpha$, and hence the constrained
MLE of $\theta$ is $\hat{\theta}_{\phi}=\left(\phi, \hat{\alpha}_{\phi}\right)^{\prime}$ which can be obtained by solving

$$
\left.\frac{\partial \ell(\theta)}{\partial \alpha}\right|_{\theta=\hat{\theta}_{\phi}}=0 .
$$

Hence, $r(\psi)$ can be calculated from (3). Moreover,

$$
\begin{aligned}
V^{\prime} & =\left.\frac{\partial y^{\prime}}{\partial \theta}\right|_{\left(y^{0}, \hat{\theta}\right)}=\left(\left.\begin{array}{llll}
\frac{\partial y_{1}}{\partial \phi} & \frac{\partial y_{2}}{\partial \phi} & \cdots & \frac{\partial y_{n}}{\partial \phi} \\
\frac{\partial y_{1}}{\partial \alpha} & \frac{\partial y_{2}}{\partial \alpha} & \cdots & \left.\frac{\partial y_{n}}{\partial \alpha}\right)
\end{array}\right|_{\left(y^{0}, \hat{\theta}\right)}=\left(\begin{array}{cccc}
y_{0} & y_{1} & \cdots & y_{n-1} \\
\frac{y_{0}^{2}\left(y_{1}-\hat{\phi} y_{0}\right)}{2\left(\omega+\hat{\alpha} y_{0}^{2}\right)} & \frac{y_{1}^{2}\left(y_{2}-\hat{\phi} y_{1}\right)}{2\left(\omega+\hat{\alpha} y_{1}^{2}\right)} & \cdots & \frac{y_{n-1}^{2}\left(y_{n}-\hat{\phi} y_{n-1}\right)}{2\left(\omega+\hat{\alpha} y_{n-1}^{2}\right)}
\end{array}\right)\right. \\
& =\left(\begin{array}{llll}
y_{0} & y_{1} & \cdots & y_{n-1} \\
v_{0} & v_{1} & \cdots & v_{n-1}
\end{array}\right) .
\end{aligned}
$$

For $1 \leq t \leq n-1$,

and for $t=n$,

$$
\begin{aligned}
& \left.\frac{\partial \ell(\theta)}{\partial y_{t}}\right|_{y^{0}}=\frac{\alpha y_{t}\left(y_{t+1}-\phi y_{t}\right)^{2}}{\left(\omega+\alpha y_{t}^{2}\right)^{2}}+\frac{\phi y_{t+1}-\left(\phi^{2}+\alpha\right) y_{t}}{\omega+\alpha y_{t}^{2}} \\
& -\frac{y_{t}-\phi y_{t-1}}{\omega+\alpha y_{t-1}^{2}} \\
& \left.\frac{\partial \ell(\theta)}{\partial y_{n}}\right|_{y^{0}}=-\frac{y_{n}-\phi y_{n-1}}{\omega+\alpha y_{n-1}^{2}} \\
& \varphi_{1}(\theta)=\sum_{t=1}^{n-1}\left\{\frac{\alpha y_{t} y_{t-1}\left(y_{t+1}-\phi y_{t}\right)^{2}}{\left(\omega+\alpha y_{t}^{2}\right)^{2}}+\frac{\phi y_{t-1} y_{t+1}-\left(\phi^{2}+\alpha\right) y_{t} y_{t-1}}{\omega+\alpha y_{t}^{2}}\right\}-\sum_{t=1}^{n-1} \frac{y_{t} y_{t-1}-\phi y_{t-1}^{2}}{\omega+\alpha y_{t-1}^{2}} \\
& =\sum_{t=1}^{n-1}\left\{\frac{\alpha y_{t} y_{t-1}\left(y_{t+1}-\phi y_{t}\right)^{2}}{\left(\omega+\alpha y_{t}^{2}\right)^{2}}+\frac{\phi y_{t-1} y_{t+1}-\left(\phi^{2}+\alpha\right) y_{t} y_{t-1}-y_{t+1} y_{t}+\phi y_{t}^{2}}{\omega+\alpha y_{t}^{2}}\right\}
\end{aligned}
$$

Then, the locally defined canonical parameter 


$$
\begin{aligned}
\varphi_{2}(\theta) & =\sum_{t=1}^{n-1}\left\{\frac{\alpha y_{t} v_{t-1}\left(y_{t+1}-\phi y_{t}\right)^{2}}{\left(\omega+\alpha y_{t}^{2}\right)^{2}}+\frac{\phi v_{t-1} y_{t+1}-\left(\phi^{2}+\alpha\right) y_{t} v_{t-1}}{\omega+\alpha y_{t}^{2}}\right\}-\sum_{t=1}^{n-1} \frac{y_{t} v_{t-1}-\phi y_{t-1} v_{t-1}}{\omega+\alpha y_{t-1}^{2}} \\
& =\sum_{t=1}^{n-1}\left\{\frac{\alpha y_{t} v_{t-1}\left(y_{t+1}-\phi y_{t}\right)^{2}}{\left(\omega+\alpha y_{t}^{2}\right)^{2}}+\frac{\phi v_{t-1} y_{t+1}-\left(\phi^{2}+\alpha\right) y_{t} v_{t-1}-y_{t+1} v_{t}+\phi y_{t} v_{t}}{\omega+\alpha y_{t}^{2}}\right\} .
\end{aligned}
$$

Thus $Q$ can be obtained from (7) with $\psi_{\theta^{\prime}}(\theta)=\frac{\partial \psi(\theta)}{\partial \theta^{\prime}}=\left(\frac{\partial \psi(\theta)}{\partial \varphi}, \frac{\partial \psi(\theta)}{\partial \alpha}\right)=(1,0)$. Finally, the modified signed log conditional likelihood ratio statistic $r^{*}(\psi)$ can be calculated from (5) and, therefore, a $(1-\gamma) 100 \%$ confidence interval for $\psi$ can be obtained from (6).

On the other hand, when the parameter of interest is $\psi(\theta)=\alpha$, the nuisance parameter is $\lambda=\phi$. The constrained MLE of $\theta$ is $\hat{\theta}_{\alpha}=\left(\hat{\phi}_{\alpha}, \alpha\right)^{\prime}$ which can be obtained by solving

$$
\left.\frac{\partial \ell(\theta)}{\partial \phi}\right|_{\theta=\hat{\theta}_{\alpha}}=0 .
$$

Again $r(\psi)$ can be calculated from (3). The tangent direction $V$ remains unchanged as above and hence $Q$ can be obtained from (7) with $\psi_{\theta^{\prime}}(\theta)=\frac{\partial \psi(\theta)}{\partial \theta^{\prime}}=\left(\frac{\partial \psi(\theta)}{\partial \varphi}, \frac{\partial \psi(\theta)}{\partial \alpha}\right)=(0,1)$. And once again, the modified signed log conditional likelihood ratio statistic $r^{*}(\psi)$ can be calculated from (5) and therefore a $(1-\gamma) 100 \%$ confidence interval for $\psi$ can be obtained from (6).

\section{Numerical Studies}

Anderson [13] considered the closing prices of the Imperial Chemical Industries (I.C.I.) for the period 25 August, 1972 to 19 January, 1973. Instead of using the raw data, we use the calibrated data, which is obtained by taking the difference of two consecutive data points after a log transformation. A scatter plot for the calibrated data is shown in Figure 2.

The DAR(1) model is employed. By applying Ling \& Li [12], we have an estimate of $\omega$ being 0.0002 . The overall MLE is obtained by maximizing (8) and we have $\hat{\theta}$ is $(-0.1553,0.1330)^{\prime}$. Table 3 reports the $90 \%$ con-

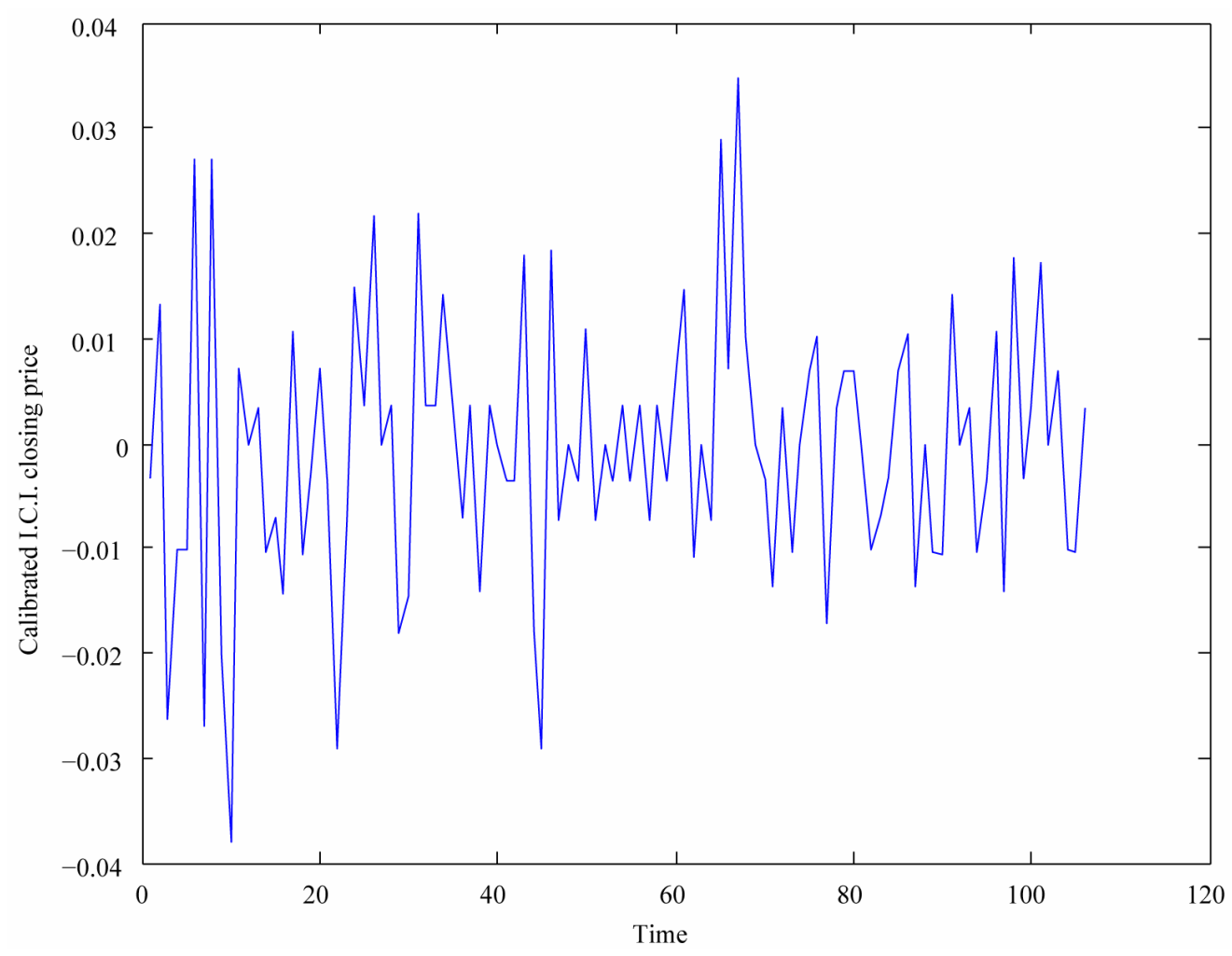

Figure 2. Scatter plot for calibrated data. 
Table $3.90 \%$ central confidence intervals for $\phi$ and $\alpha$.

\begin{tabular}{ccc}
\hline Method 90\% Confidence Interval for $\phi$ & $90 \%$ Confidence Interval for $\alpha$ \\
\hline BN & $(-0.3629,0.0389)$ & $(0.0048,0.4719)$ \\
SLR & $(-0.3490,0.0352)$ & $(0.0000,0.4072)$ \\
Ling & $(-0.3438,0.0333)$ & $(0.0048,0.3588)$ \\
\hline
\end{tabular}

fidence intervals for $\phi$, and the $90 \%$ confidence intervals for $\alpha$, obtained from the methods discussed in this paper: BN is the proposed method based on Equation (5), SLR is the signed log-likelihood ratio statistic (3), Ling is the method discussed in Ling [5]. SLR and Ling give similar confidence intervals which are quite different from the results obtained by $\mathrm{BN}$. Moreover, since $\alpha$ is bounded by 0 , both SLR and Ling have deficiency on the left boundary.

The $p$-value functions are presented in Figures $\mathbf{3}$ and $\mathbf{4}$. Additionally, the two horizontal lines indicate upper and lower 0.05 levels respectively. The plots show that results obtained by the three methods discussed in this paper are quite different.

To examine the accuracy of the methods discussed in this paper, Monte Carlo simulation studies are performed. For each combination of $(\phi, \alpha), 10,000$ Monte Carlo samples, for each of sample sizes takeing values $n=50$, 200 and 400, are generated from the DAR(1) model where $\eta_{t} \sim N(0,1), t=0, \cdots, n$. Without loss of gener- ality, $\omega$ is set to be 1 .

Tables 4-9 recorded the central coverage probability (CCP) which is the proportion of intervals that contains the true $\psi$, the lower error probability (L) which is the proportion of true $\psi$ that falls outside the lower bound of the confidence interval, and upper error probability (U) which is the proportion of true $\psi$ that falls outside the upper bound of the confidence interval. The nominal values for the central coverage probability, and the lower and upper errors probabilities are $0.90,0.05$, and 0.05 respectively. In additional to this, we also report the average bias (Avg Bias) defined by $\frac{|\mathrm{L}-0.05|+|\mathrm{U}-0.05|}{2}$, which has the nominal value of 0 .

For both $\psi=\phi$ and $\psi=\alpha$, simulation studies show that $\mathrm{BN}$ is remarkably accurate even when the sample size is small. As $n$ increases, there is significant improvement on the precision of both the SLR method and the Ling's method in terms of both cental coverage and average bias but the results are still not as accurate as those obtained by the proposed method.

\section{Conclusion}

A conditional likelihood based method is proposed to obtain confidence intervals for $\alpha$ and for $\phi$ of the weak stationary DAR(1) model. Theoretically, the pro-

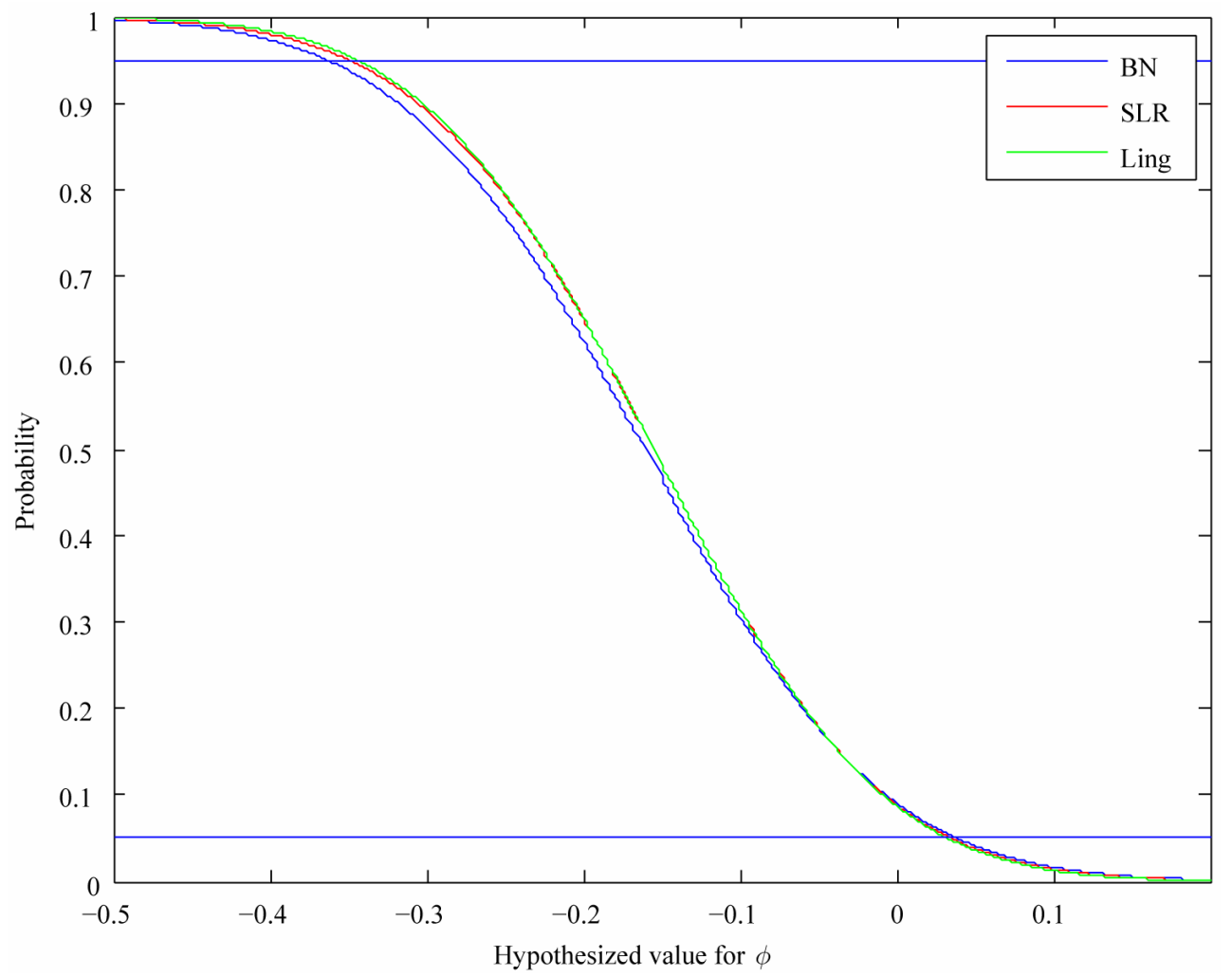

Figure 3. $p$-value functions for $\phi$ of I.C.I. Data. 


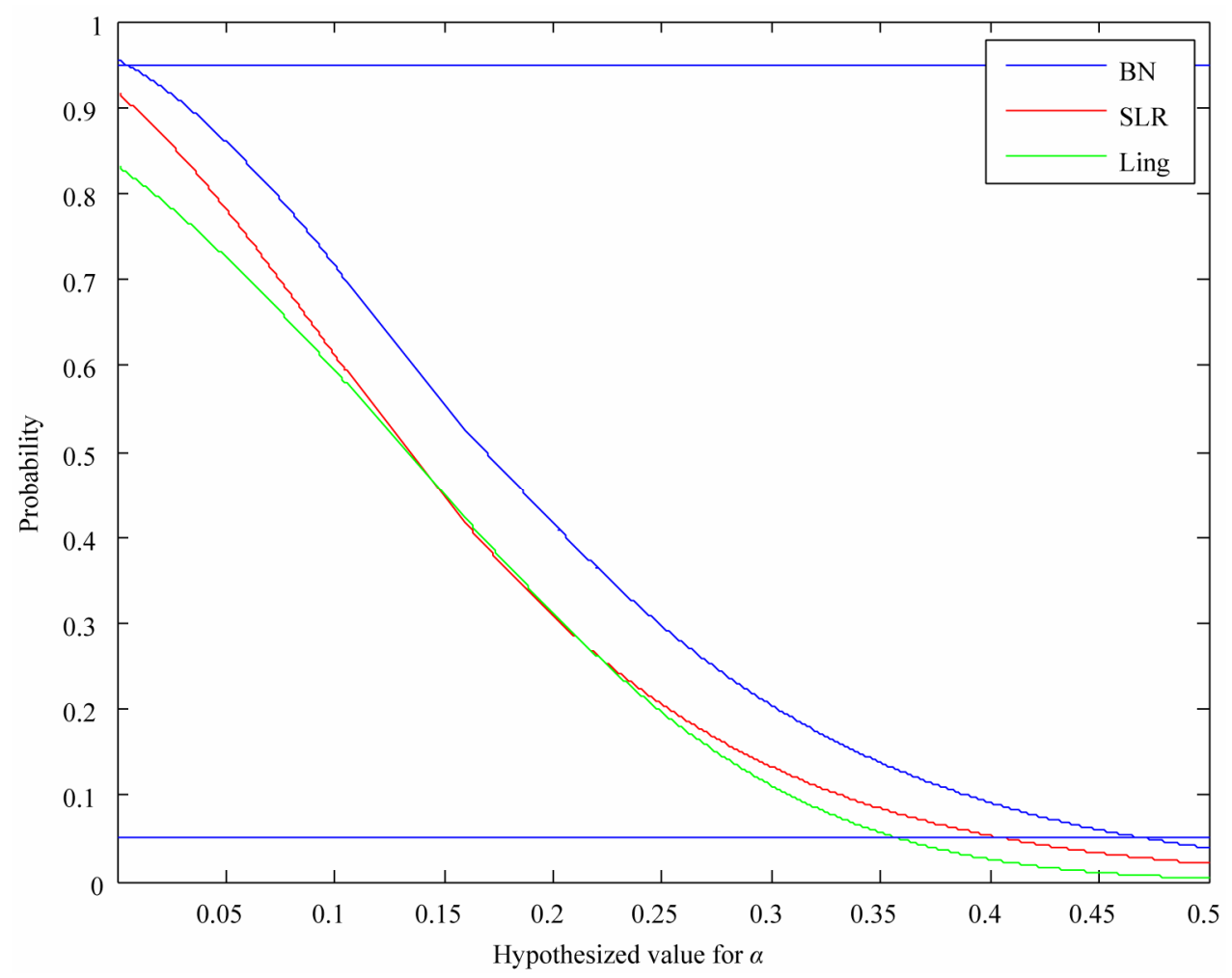

Figure 4. $p$-value functions for $\alpha$ of I.C.I. Data.

Table 4. Simulation results for some boundary points of the DAR(1) model when $\boldsymbol{n}=\mathbf{5 0}$.

\begin{tabular}{|c|c|c|c|c|c|c|c|c|c|c|}
\hline & & & $\psi=\phi$ & & & & $\psi=\alpha$ & & & \\
\hline$\phi$ & $\alpha$ & Method & $\mathrm{L}$ & $\mathrm{U}$ & СCP & Avg Bias & $\mathrm{L}$ & $\mathrm{U}$ & СCP & Avg Bias \\
\hline \multirow[t]{3}{*}{-0.95} & 0.0975 & $\mathrm{BN}$ & 0.0544 & 0.0451 & 0.9005 & 0.0046 & 0.0372 & 0.0573 & 0.9055 & 0.0101 \\
\hline & & SLR & 0.0343 & 0.0760 & 0.8897 & 0.0209 & 0.0870 & 0.0314 & 0.8816 & 0.0278 \\
\hline & & Ling & 0.0384 & 0.0924 & 0.8692 & 0.0270 & 0.1228 & 0.0020 & 0.8752 & 0.0604 \\
\hline \multirow[t]{3}{*}{-0.5} & 0.75 & $\mathrm{BN}$ & 0.0542 & 0.0494 & 0.8964 & 0.0024 & 0.0542 & 0.0494 & 0.8964 & 0.0024 \\
\hline & & SLR & 0.0950 & 0.0272 & 0.8778 & 0.0339 & 0.0950 & 0.0272 & 0.8778 & 0.0339 \\
\hline & & Ling & 0.1558 & 0.0041 & 0.8401 & 0.0759 & 0.1558 & 0.0041 & 0.8401 & 0.0759 \\
\hline \multirow[t]{3}{*}{0} & 1 & $\mathrm{BN}$ & 0.0521 & 0.0529 & 0.8950 & 0.0025 & 0.0497 & 0.0524 & 0.8979 & 0.0014 \\
\hline & & SLR & 0.0560 & 0.0571 & 0.8869 & 0.0065 & 0.0987 & 0.0275 & 0.8738 & 0.0356 \\
\hline & & Ling & 0.0625 & 0.0645 & 0.8730 & 0.0135 & 0.1532 & 0.0044 & 0.8424 & 0.0744 \\
\hline \multirow[t]{3}{*}{0.5} & 0.75 & $\mathrm{BN}$ & 0.0487 & 0.0506 & 0.9007 & 0.0009 & 0.0536 & 0.0492 & 0.8972 & 0.0022 \\
\hline & & SLR & 0.0630 & 0.0491 & 0.8879 & 0.0070 & 0.0964 & 0.0264 & 0.8772 & 0.0350 \\
\hline & & Ling & 0.0745 & 0.0565 & 0.8690 & 0.0155 & 0.1545 & 0.0026 & 0.8429 & 0.0760 \\
\hline \multirow[t]{3}{*}{0.95} & 0.0975 & $\mathrm{BN}$ & 0.0446 & 0.0576 & 0.8978 & 0.0065 & 0.0375 & 0.0542 & 0.9083 & 0.0083 \\
\hline & & SLR & 0.0761 & 0.0369 & 0.8870 & 0.0196 & 0.0856 & 0.0331 & 0.8813 & 0.0262 \\
\hline & & Ling & 0.0893 & 0.0408 & 0.8699 & 0.0243 & 0.1223 & 0.0014 & 0.8763 & 0.0605 \\
\hline
\end{tabular}


Table 5. Simulation results for some interior points of the DAR(1) $\operatorname{model}$ when $\boldsymbol{n}=\mathbf{5 0}$.

\begin{tabular}{|c|c|c|c|c|c|c|c|c|c|c|}
\hline & & & $\psi=\phi$ & & & & $\psi=\alpha$ & & & \\
\hline$\phi$ & $\alpha$ & Method & $\mathrm{L}$ & $\mathrm{U}$ & ССР & Avg Bias & $\mathrm{L}$ & $\mathrm{U}$ & ССР & Avg Bias \\
\hline \multirow[t]{3}{*}{-0.95} & 0.09 & $\mathrm{BN}$ & 0.0574 & 0.0435 & 0.8991 & 0.0070 & 0.0322 & 0.0533 & 0.9145 & 0.0106 \\
\hline & & SLR & 0.0363 & 0.0734 & 0.8903 & 0.0186 & 0.0838 & 0.0290 & 0.8872 & 0.0274 \\
\hline & & Ling & 0.0399 & 0.0880 & 0.8721 & 0.0240 & 0.1161 & 0.0017 & 0.8822 & 0.0572 \\
\hline \multirow[t]{3}{*}{-0.5} & 0.5 & $\mathrm{BN}$ & 0.0536 & 0.0483 & 0.8981 & 0.0026 & 0.0497 & 0.0524 & 0.8979 & 0.0014 \\
\hline & & SLR & 0.0508 & 0.0606 & 0.8886 & 0.0057 & 0.1078 & 0.0291 & 0.8631 & 0.0394 \\
\hline & & Ling & 0.0569 & 0.0702 & 0.8729 & 0.0135 & 0.1724 & 0.0032 & 0.8244 & 0.0846 \\
\hline \multirow[t]{3}{*}{-0.5} & 0.25 & $\mathrm{BN}$ & 0.0507 & 0.0488 & 0.9005 & 0.0009 & 0.0113 & 0.0514 & 0.9373 & 0.0201 \\
\hline & & SLR & 0.0467 & 0.0598 & 0.8935 & 0.0066 & 0.0997 & 0.0249 & 0.8754 & 0.0374 \\
\hline & & Ling & 0.0527 & 0.0686 & 0.8787 & 0.0106 & 0.1522 & 0.0016 & 0.8462 & 0.0753 \\
\hline \multirow[t]{3}{*}{0} & 0.5 & $\mathrm{BN}$ & 0.0507 & 0.0499 & 0.8994 & 0.0004 & 0.0391 & 0.0542 & 0.9067 & 0.0075 \\
\hline & & SLR & 0.0552 & 0.0535 & 0.8913 & 0.0043 & 0.1077 & 0.0279 & 0.8644 & 0.0399 \\
\hline & & Ling & 0.0623 & 0.0612 & 0.8765 & 0.0117 & 0.1750 & 0.0027 & 0.8223 & 0.0861 \\
\hline \multirow[t]{3}{*}{0.1} & 0.4 & $\mathrm{BN}$ & 0.0507 & 0.0506 & 0.8987 & 0.0006 & 0.0201 & 0.0538 & 0.9261 & 0.0169 \\
\hline & & SLR & 0.0552 & 0.0524 & 0.8924 & 0.0038 & 0.1078 & 0.0259 & 0.8663 & 0.0410 \\
\hline & & Ling & 0.0639 & 0.0580 & 0.8781 & 0.0109 & 0.1802 & 0.0012 & 0.8186 & 0.0895 \\
\hline \multirow[t]{3}{*}{0.5} & 0.25 & $\mathrm{BN}$ & 0.0490 & 0.0481 & 0.9029 & 0.0015 & 0.0125 & 0.0518 & 0.9357 & 0.0197 \\
\hline & & SLR & 0.0601 & 0.0445 & 0.8954 & 0.0078 & 0.1034 & 0.0279 & 0.8687 & 0.0378 \\
\hline & & Ling & 0.0709 & 0.0512 & 0.8779 & 0.0111 & 0.1657 & 0.0013 & 0.8330 & 0.0822 \\
\hline \multirow[t]{3}{*}{0.5} & 0.5 & $\mathrm{BN}$ & 0.0488 & 0.0472 & 0.9040 & 0.0020 & 0.0459 & 0.0566 & 0.8975 & 0.0053 \\
\hline & & SLR & 0.0627 & 0.0453 & 0.8920 & 0.0087 & 0.1016 & 0.0296 & 0.8688 & 0.0360 \\
\hline & & Ling & 0.0731 & 0.0524 & 0.8745 & 0.0127 & 0.1651 & 0.0028 & 0.8321 & 0.0812 \\
\hline \multirow[t]{3}{*}{0.95} & 0.09 & $\mathrm{BN}$ & 0.0457 & 0.0538 & 0.9005 & 0.0041 & 0.0342 & 0.0531 & 0.9127 & 0.0095 \\
\hline & & SLR & 0.0783 & 0.0344 & 0.8873 & 0.0219 & 0.0847 & 0.0306 & 0.8847 & 0.0270 \\
\hline & & Ling & 0.0928 & 0.0386 & 0.8686 & 0.0271 & 0.1166 & 0.0023 & 0.8811 & 0.0571 \\
\hline
\end{tabular}

Table 6. Simulation results for some boundary points of the DAR(1) model when $n=200$.

\begin{tabular}{|c|c|c|c|c|c|c|c|c|c|c|}
\hline & & & $\psi=\phi$ & & & & $\psi=\alpha$ & & & \\
\hline$\phi$ & $\alpha$ & Method & $\mathrm{L}$ & $\mathrm{U}$ & CCP & Avg Bias & $\mathrm{L}$ & $\mathrm{U}$ & CCP & Avg Bias \\
\hline \multirow[t]{3}{*}{-0.95} & 0.0975 & $\mathrm{BN}$ & 0.0552 & 0.0516 & 0.8932 & 0.0034 & 0.0622 & 0.0498 & 0.8880 & 0.0062 \\
\hline & & SLR & 0.0415 & 0.0623 & 0.8962 & 0.0104 & 0.0699 & 0.0398 & 0.8903 & 0.0151 \\
\hline & & Ling & 0.0427 & 0.0655 & 0.8918 & 0.0114 & 0.0832 & 0.0093 & 0.9075 & 0.0369 \\
\hline \multirow[t]{3}{*}{-0.5} & 0.75 & $\mathrm{BN}$ & 0.0519 & 0.0509 & 0.8972 & 0.0014 & 0.0485 & 0.0521 & 0.8994 & 0.0018 \\
\hline & & SLR & 0.0506 & 0.0534 & 0.8960 & 0.0020 & 0.0641 & 0.0361 & 0.8998 & 0.0140 \\
\hline & & Ling & 0.0521 & 0.0551 & 0.8928 & 0.0036 & 0.0769 & 0.0010 & 0.9221 & 0.0379 \\
\hline \multirow[t]{3}{*}{0} & 1 & $\mathrm{BN}$ & 0.0517 & 0.0514 & 0.8969 & 0.0015 & 0.0522 & 0.0515 & 0.8963 & 0.0019 \\
\hline & & SLR & 0.0527 & 0.0528 & 0.8945 & 0.0027 & 0.0686 & 0.0381 & 0.8933 & 0.0153 \\
\hline & & Ling & 0.0515 & 0.0520 & 0.8965 & 0.0017 & 0.1049 & 0.0208 & 0.8743 & 0.0421 \\
\hline \multirow[t]{3}{*}{0.5} & 0.75 & $\mathrm{BN}$ & 0.0534 & 0.0500 & 0.8966 & 0.0017 & 0.0472 & 0.0545 & 0.8983 & 0.0037 \\
\hline & & SLR & 0.0557 & 0.0494 & 0.8949 & 0.0032 & 0.0674 & 0.0388 & 0.8938 & 0.0143 \\
\hline & & Ling & 0.0580 & 0.0504 & 0.8916 & 0.0042 & 0.0777 & 0.0009 & 0.9214 & 0.0384 \\
\hline \multirow[t]{3}{*}{0.95} & 0.0975 & $\mathrm{BN}$ & 0.0576 & 0.0499 & 0.8925 & 0.0038 & 0.0597 & 0.0503 & 0.8900 & 0.0050 \\
\hline & & SLR & 0.0677 & 0.0384 & 0.8939 & 0.0147 & 0.0653 & 0.0379 & 0.8968 & 0.0137 \\
\hline & & Ling & 0.0709 & 0.0396 & 0.8895 & 0.0157 & 0.0787 & 0.0097 & 0.9116 & 0.0345 \\
\hline
\end{tabular}


Table 7. Simulation results for some interior points of the DAR(1) $\operatorname{model}$ when $\boldsymbol{n}=\mathbf{2 0 0}$.

\begin{tabular}{|c|c|c|c|c|c|c|c|c|c|c|}
\hline & & & $\psi=\phi$ & & & & $\psi=\alpha$ & & & \\
\hline$\phi$ & $\alpha$ & Method & $\mathrm{L}$ & $\mathrm{U}$ & ССР & Avg Bias & $\mathrm{L}$ & $\mathrm{U}$ & CCP & Avg Bias \\
\hline \multirow[t]{3}{*}{-0.95} & 0.09 & $\mathrm{BN}$ & 0.0509 & 0.0512 & 0.8979 & 0.0010 & 0.0585 & 0.0529 & 0.8886 & 0.0057 \\
\hline & & SLR & 0.0401 & 0.0641 & 0.8958 & 0.0120 & 0.0706 & 0.0390 & 0.8904 & 0.0158 \\
\hline & & Ling & 0.0411 & 0.0663 & 0.8926 & 0.0126 & 0.0844 & 0.0091 & 0.9065 & 0.0377 \\
\hline \multirow[t]{3}{*}{-0.5} & 0.5 & $\mathrm{BN}$ & 0.0486 & 0.0512 & 0.9002 & 0.0013 & 0.0497 & 0.0469 & 0.9034 & 0.0017 \\
\hline & & SLR & 0.0478 & 0.0545 & 0.8977 & 0.0033 & 0.0659 & 0.0354 & 0.8987 & 0.0153 \\
\hline & & Ling & 0.0491 & 0.0564 & 0.8945 & 0.0037 & 0.0787 & 0.0101 & 0.9112 & 0.0343 \\
\hline \multirow[t]{3}{*}{-0.5} & 0.25 & $\mathrm{BN}$ & 0.0458 & 0.0524 & 0.9018 & 0.0033 & 0.0559 & 0.0503 & 0.8938 & 0.0031 \\
\hline & & SLR & 0.0452 & 0.0563 & 0.8985 & 0.0056 & 0.0771 & 0.0352 & 0.8877 & 0.0210 \\
\hline & & Ling & 0.0464 & 0.0595 & 0.8941 & 0.0066 & 0.0891 & 0.0057 & 0.9052 & 0.0417 \\
\hline \multirow[t]{3}{*}{0} & 0.5 & $\mathrm{BN}$ & 0.0495 & 0.0544 & 0.8961 & 0.0024 & 0.0512 & 0.0523 & 0.8965 & 0.0017 \\
\hline & & SLR & 0.0518 & 0.0568 & 0.8914 & 0.0043 & 0.0732 & 0.0378 & 0.8890 & 0.0177 \\
\hline & & Ling & 0.0623 & 0.0612 & 0.8765 & 0.0117 & 0.0808 & 0.0093 & 0.9099 & 0.0357 \\
\hline \multirow[t]{3}{*}{0.1} & 0.4 & $\mathrm{BN}$ & 0.0514 & 0.0504 & 0.8982 & 0.0009 & 0.0499 & 0.0487 & 0.9014 & 0.0007 \\
\hline & & SLR & 0.0523 & 0.0506 & 0.8971 & 0.0014 & 0.0740 & 0.0330 & 0.8930 & 0.0205 \\
\hline & & Ling & 0.0538 & 0.0519 & 0.8943 & 0.0028 & 0.0827 & 0.0073 & 0.9100 & 0.0377 \\
\hline \multirow[t]{3}{*}{0.5} & 0.25 & $\mathrm{BN}$ & 0.0478 & 0.0465 & 0.9057 & 0.0029 & 0.0537 & 0.0500 & 0.8963 & 0.0018 \\
\hline & & SLR & 0.0522 & 0.0449 & 0.9029 & 0.0037 & 0.0733 & 0.0370 & 0.8897 & 0.0182 \\
\hline & & Ling & 0.0549 & 0.0467 & 0.8984 & 0.0041 & 0.0873 & 0.0064 & 0.9063 & 0.0405 \\
\hline \multirow[t]{3}{*}{0.5} & 0.5 & $\mathrm{BN}$ & 0.0514 & 0.0472 & 0.9014 & 0.0021 & 0.0513 & 0.0462 & 0.9025 & 0.0026 \\
\hline & & SLR & 0.0537 & 0.0459 & 0.9004 & 0.0039 & 0.0665 & 0.0319 & 0.9016 & 0.0173 \\
\hline & & Ling & 0.0558 & 0.0478 & 0.8964 & 0.0040 & 0.0782 & 0.0073 & 0.9145 & 0.0355 \\
\hline \multirow[t]{3}{*}{0.95} & 0.09 & $\mathrm{BN}$ & 0.0543 & 0.0481 & 0.8976 & 0.0031 & 0.0571 & 0.0531 & 0.8898 & 0.0051 \\
\hline & & SLR & 0.0669 & 0.0364 & 0.8967 & 0.0153 & 0.0682 & 0.0383 & 0.8935 & 0.0149 \\
\hline & & Ling & 0.0691 & 0.0373 & 0.8936 & 0.0159 & 0.0820 & 0.0088 & 0.9092 & 0.0366 \\
\hline
\end{tabular}

Table 8. Simulation results for some boundary points of the DAR(1) model when $n=400$.

\begin{tabular}{|c|c|c|c|c|c|c|c|c|c|c|}
\hline & & & $\psi=\phi$ & & & & $\psi=\alpha$ & & & \\
\hline$\phi$ & $\alpha$ & Method & $\mathrm{L}$ & $\mathrm{U}$ & ССР & Avg Bias & $\mathrm{L}$ & $\mathrm{U}$ & ССР & Avg Bias \\
\hline \multirow[t]{3}{*}{-0.95} & 0.0975 & $\mathrm{BN}$ & 0.0488 & 0.0523 & 0.8989 & 0.0017 & 0.0613 & 0.0528 & 0.8859 & 0.0070 \\
\hline & & SLR & 0.0398 & 0.0599 & 0.9003 & 0.0101 & 0.0602 & 0.0415 & 0.8983 & 0.0093 \\
\hline & & Ling & 0.0404 & 0.0607 & 0.8989 & 0.0101 & 0.0635 & 0.0152 & 0.9213 & 0.0242 \\
\hline \multirow[t]{3}{*}{-0.5} & 0.75 & $\mathrm{BN}$ & 0.0531 & 0.0488 & 0.8981 & 0.0021 & 0.0503 & 0.0541 & 0.8956 & 0.0022 \\
\hline & & SLR & 0.0523 & 0.0510 & 0.8967 & 0.0016 & 0.0603 & 0.0419 & 0.8978 & 0.0092 \\
\hline & & Ling & 0.0529 & 0.0516 & 0.8955 & 0.0022 & 0.0610 & 0.0178 & 0.9212 & 0.0216 \\
\hline \multirow[t]{3}{*}{0} & 1 & $\mathrm{BN}$ & 0.0577 & 0.0513 & 0.8910 & 0.0045 & 0.0496 & 0.0512 & 0.8992 & 0.0008 \\
\hline & & SLR & 0.0563 & 0.0506 & 0.8931 & 0.0034 & 0.0611 & 0.0413 & 0.8976 & 0.0099 \\
\hline & & Ling & 0.0566 & 0.0510 & 0.8924 & 0.0038 & 0.0815 & 0.0280 & 0.8905 & 0.0268 \\
\hline \multirow[t]{3}{*}{0.5} & 0.75 & $\mathrm{BN}$ & 0.0529 & 0.0528 & 0.8943 & 0.0028 & 0.0533 & 0.0546 & 0.8921 & 0.0039 \\
\hline & & SLR & 0.0544 & 0.0514 & 0.8942 & 0.0029 & 0.0646 & 0.0411 & 0.8943 & 0.0118 \\
\hline & & Ling & 0.0550 & 0.0521 & 0.8929 & 0.0035 & 0.0655 & 0.0192 & 0.9153 & 0.0232 \\
\hline \multirow[t]{3}{*}{0.95} & 0.0975 & $\mathrm{BN}$ & 0.0524 & 0.0528 & 0.8948 & 0.0026 & 0.0634 & 0.0528 & 0.8838 & 0.0081 \\
\hline & & SLR & 0.0612 & 0.0435 & 0.8953 & 0.0089 & 0.0640 & 0.0429 & 0.8931 & 0.0106 \\
\hline & & Ling & 0.0625 & 0.0442 & 0.8933 & 0.0091 & 0.0675 & 0.0159 & 0.9166 & 0.0258 \\
\hline
\end{tabular}


Table 9. Simulation results for some interior points of the DAR(1) $\operatorname{model}$ when $\boldsymbol{n}=\mathbf{4 0 0}$.

\begin{tabular}{|c|c|c|c|c|c|c|c|c|c|c|}
\hline & & & $\psi=\phi$ & & & & $\psi=\alpha$ & & & \\
\hline$\phi$ & $\alpha$ & Method & $\mathrm{L}$ & $\mathrm{U}$ & ССР & Avg Bias & $\mathrm{L}$ & $\mathrm{U}$ & ССР & Avg Bias \\
\hline \multirow[t]{3}{*}{-0.95} & 0.09 & $\mathrm{BN}$ & 0.0481 & 0.0519 & 0.9000 & 0.0019 & 0.0529 & 0.0551 & 0.8920 & 0.0040 \\
\hline & & SLR & 0.0402 & 0.0594 & 0.9004 & 0.0096 & 0.0569 & 0.0407 & 0.9024 & 0.0081 \\
\hline & & Ling & 0.0404 & 0.0603 & 0.8993 & 0.0100 & 0.0611 & 0.0159 & 0.9230 & 0.0226 \\
\hline \multirow[t]{3}{*}{-0.5} & 0.5 & $\mathrm{BN}$ & 0.0540 & 0.0506 & 0.8954 & 0.0023 & 0.0520 & 0.0471 & 0.9009 & 0.0024 \\
\hline & & SLR & 0.0526 & 0.0520 & 0.8954 & 0.0023 & 0.0622 & 0.0384 & 0.8994 & 0.0119 \\
\hline & & Ling & 0.0537 & 0.0538 & 0.8925 & 0.0037 & 0.0623 & 0.0133 & 0.9244 & 0.0245 \\
\hline \multirow[t]{3}{*}{-0.5} & 0.25 & $\mathrm{BN}$ & 0.0529 & 0.0502 & 0.8969 & 0.0015 & 0.0549 & 0.0524 & 0.8927 & 0.0036 \\
\hline & & SLR & 0.0514 & 0.0525 & 0.8961 & 0.0019 & 0.0652 & 0.0403 & 0.8945 & 0.0124 \\
\hline & & Ling & 0.0521 & 0.0533 & 0.8946 & 0.0027 & 0.0634 & 0.0105 & 0.9261 & 0.0264 \\
\hline \multirow[t]{3}{*}{0} & 0.5 & $\mathrm{BN}$ & 0.0539 & 0.0511 & 0.8950 & 0.0025 & 0.0528 & 0.0463 & 0.9009 & 0.0032 \\
\hline & & SLR & 0.0537 & 0.0513 & 0.8950 & 0.0025 & 0.0636 & 0.0353 & 0.9011 & 0.0142 \\
\hline & & Ling & 0.0544 & 0.0524 & 0.8932 & 0.0034 & 0.0613 & 0.0109 & 0.9278 & 0.0252 \\
\hline \multirow[t]{3}{*}{0.1} & 0.4 & $\mathrm{BN}$ & 0.0549 & 0.0538 & 0.8913 & 0.0043 & 0.0499 & 0.0568 & 0.8933 & 0.0035 \\
\hline & & SLR & 0.0548 & 0.0533 & 0.8919 & 0.0040 & 0.0641 & 0.0411 & 0.8948 & 0.0115 \\
\hline & & Ling & 0.0559 & 0.0544 & 0.8897 & 0.0051 & 0.0610 & 0.0126 & 0.9264 & 0.0242 \\
\hline \multirow[t]{3}{*}{0.5} & 0.25 & $\mathrm{BN}$ & 0.0530 & 0.0485 & 0.8985 & 0.0022 & 0.0563 & 0.0497 & 0.8940 & 0.0033 \\
\hline & & SLR & 0.0545 & 0.0474 & 0.8981 & 0.0036 & 0.0679 & 0.0382 & 0.8939 & 0.0149 \\
\hline & & Ling & 0.0556 & 0.0487 & 0.8957 & 0.0034 & 0.0648 & 0.0113 & 0.9239 & 0.0267 \\
\hline \multirow[t]{3}{*}{0.5} & 0.5 & $\mathrm{BN}$ & 0.0521 & 0.0476 & 0.9003 & 0.0022 & 0.0545 & 0.0502 & 0.8953 & 0.0023 \\
\hline & & SLR & 0.0535 & 0.0466 & 0.8999 & 0.0034 & 0.0638 & 0.0401 & 0.8961 & 0.0118 \\
\hline & & Ling & 0.0542 & 0.0472 & 0.8986 & 0.0035 & 0.0637 & 0.0141 & 0.9222 & 0.0248 \\
\hline \multirow[t]{3}{*}{0.95} & 0.09 & $\mathrm{BN}$ & 0.0515 & 0.0537 & 0.8948 & 0.0026 & 0.0526 & 0.0571 & 0.8903 & 0.0048 \\
\hline & & SLR & 0.0594 & 0.0424 & 0.8982 & 0.0085 & 0.0577 & 0.0453 & 0.8970 & 0.0062 \\
\hline & & Ling & 0.0605 & 0.0434 & 0.8961 & 0.0085 & 0.0608 & 0.0171 & 0.9221 & 0.0218 \\
\hline
\end{tabular}

posed method has rate of convergence $O\left(n^{-3 / 2}\right)$. The simulation results have indicated that the proposed method significantly improves the accuracy of the inference over some existing methods. All the above discussed methods are implemented in MATLAB and the code is available from the first author.

\section{REFERENCES}

[1] R. F. Engle, “Autoregressive Conditional Heteroscedasticity with Estimates of the Variance of Inflationary Expectations,” Econometrica, Vol. 50, 1982, pp. 987-1007. doi:10.2307/1912773

[2] A. A. Weiss, "ARMA Models with ARCH Errors," Journal of Time Series Analysis, Vol. 3, 1984, pp. 129-143. doi:10.1111/j.1467-9892.1984.tb00382.x

[3] D. Guégan and J. Diebolt, "Probabilistic Properties of the $\beta$-ARCH-Model,” Statistica Sininca, Vol. 4, 1994, pp. 71-87.

[4] M. Borkovec and C. Klüppelberg, "The Tail of the Stationary Distribution of an Autoregressive Process with ARCH(1) Errors," Annals of Applied Probability, Vol. 11,
No. 4, 1998, pp. 1220-1241.

[5] S. Ling, "Estimation and Testing Stationarity for Double-Auto Regressive Models," Journal of the Royal Statistical Society Series B, Vol. 66, No. 1, 2004, pp. 63-78. doi:10.1111/j.1467-9868.2004.00432.x

[6] A. Wald, “Tests of Statistical Hypotheses Concerning Several Parameters When the Number of Observations Is Large," Transactions of the American Mathematical Society, Vol. 54, No. 3, 1943, pp. 426-482. doi:10.1090/S0002-9947-1943-0012401-3

[7] T. Di Ciccio, C. Field and D. A. S. Fraser, “Approxmation of Marginal Tail Probabilities and Inference for Scalar Parameters,” Biometrika, Vol. 77, No. 1, 1990, pp. 77-95. doi:10.1093/biomet/77.1.77

[8] O. E. Barndorff-Nielsen, "Inference on Full and Partial Parameters Based on the Standardized Signed Log-likelihood Ratio,” Biometrika, Vol. 73, 1986, pp. 307-322.

[9] O. E. Barndorff-Nielsen, "Modified Signed Log-Likelihood Ratio Statistic,” Biometrika, Vol. 78, No. 3, 1991, pp. 557-563. doi:10.1093/biomet/78.3.557

[10] D. A. S. Fraser and N. Reid, "Ancillaries and Third-Order Significance,” Utilitas Mathematica, Vol. 47, 1995, pp. 
33-53.

[11] D. Ling, “A Double AR(p) Model: Structure and Estimation,” Statistica Sinica, Vol. 17, 2007, pp. 161-175.

[12] S. Ling and D. Li, “Asymptotic Inference for a Nonsta- tionary Double AR(1) Model,” Biometrika, Vol. 95, No. 1, 2008, pp. 257-263. doi:10.1093/biomet/asm084

[13] O. D. Anderson, "Time Series Analysis and Forecasting: The Box-Jenkins Approach,” Butterworth, 1976. 